A. Weisbecker, M. Burmester \& A. Schmidt (Hrsg.): Mensch und Computer 2015 Workshopband, Stuttgart: Oldenbourg Wissenschaftsverlag, 2015, S. 617-619.

\title{
Usable Security and Privacy: Nutzerzentrierte Lösungsansätze zum Schutz sensibler Daten
}

\author{
Luigi Lo Iacono ${ }^{1}$, Hartmut Schmitt ${ }^{2}$ \\ Fachhochschule Köln ${ }^{1}$ \\ HK Business Solutions $\mathrm{GmbH}^{2}$
}

\section{Thema}

Die digitale Vernetzung und die zunehmende Technologisierung unseres beruflichen und privaten Alltags stellen neue Herausforderungen an den Schutz sensibler Daten. Damit sich Software, Apps und interaktive Produkte im täglichen Gebrauch nicht als Risiken für private Endanwender oder Unternehmen entpuppen, müssen sie mit Sicherheitsfunktionen und mechanismen ausgestattet sein, die von allen Nutzergruppen verstanden und benutzt werden können.

Das Problem: Bei der Nutzung von Software und interaktiven Produkten zählen Datenschutz und -sicherheit in der Regel nicht $\mathrm{zu}$ den primären Zielen des Anwenders. Die Entwicklungsprozesse und Vorgehensmodelle des Usability-Engineerings und des UserCentered Design sind daher noch weitgehend von denen des Security-Engineerings entkoppelt. Um die Grundlage für eine systematische Entwicklung sicherer und benutzbarer Produkte zu schaffen, müssen also die Konzepte, Methoden und Werkzeuge verschiedener Software-Engineering-Disziplinen und Geisteswissenschaften miteinander integriert werden und neue nutzerzentrierte Lösungsansätze entwickelt werden.

Usable Security and Privacy bezeichnet den inter- und transdisziplinären Ansatz, sicherheitsfördernde Verfahren für Software und interaktive Produkte so zu gestalten, dass Benutzer bei ihren sicherheits- und datenschutzrelevanten Zielen und Vorhaben bestmöglich unterstützt werden. Hierdurch werden z. B. auch Laien und technikferne Anwender in die Lage versetzt, Sicherheitselemente und deren Notwendigkeit zumindest grundlegend zu verstehen und diese in der dafür vorgesehenen Weise zu verwenden. 


\section{Zielsetzung und Inhalte des Workshops}

Mit dem Workshop „Usable Security and Privacy: Nutzerzentrierte Lösungsansätze zum Schutz sensibler Daten" soll ein Forum etabliert werden, in dem sich Experten aus Wissenschaft und Praxis zum Thema benutzerfreundliche Informationssicherheit austauschen können. Dies umfasst die Präsentation aktueller Forschungsarbeiten und (prototypischer) Entwicklungen, den wissenschaftlichen Diskurs, die interdisziplinäre Auseinandersetzung und den praktischen Erfahrungsaustausch zwischen den Teilnehmern. Zugleich soll durch den Workshop die Diskussion für ein breiteres Fachpublikum geöffnet werden und es sollen neue Impulse für das Forschungsgebiet Usable Security and Privacy gesetzt werden, z. B. indem Fragestellungen für künftige Forschungsarbeiten aufgeworfen werden.

Das Thema Usable Security and Privacy betrifft unterschiedliche Forschungsgebiete und Berufsfelder, z. B. Informatik, Ingenieurwissenschaften, Mediengestaltung und Psychologie. Der Workshop richtet sich daher an interessierte Wissenschaftler aus diesen Bereichen, aber auch ausdrücklich an Praktiker aus der Wirtschaft und der Industrie.

Erstmals wurde der Workshop im Rahmen der Fachtagung Mensch und Computer 2015 als ganztägige Veranstaltung am 6.9.2015 in Stuttgart durchgeführt. Er hatte zum Ziel, neue nutzerzentrierte Ansätze zum Schutz sensibler Daten zu thematisieren und zu diskutieren, um so den Dialog zwischen Wissenschaft und Industrie anzuregen. Mögliche Themen für wissenschaftliche oder praxisorientierte Beiträge des Workshops waren:

- neue Vorgehensweisen oder Werkzeuge,

- gestalterische Studien,

- Berichte praktischer Umsetzung (erfolgreiche/fehlgeschlagene Beispiele),

- Systemdemonstrationen,

- praxiserprobte Methoden, Best Practices,

- kritische Reflexionen (Herausforderungen, Fallstricke) und

- theoretische/zukunftsweisende Arbeiten.

Sämtliche angenommenen Beiträge wurden in Vorträgen vorgestellt und diskutiert. Ergebnis des Workshops ist die hier vorgestellte Sammlung von neuen Entwicklungen und Forschungsergebnissen im Bereich Usable Security and Privacy.

\section{Programmkomitee}

Das Programmkomitee des Workshops übernahm die fachliche und inhaltliche Begutachtung der Einreichungen und unterstützte die Verbreitung des Calls for Papers. Die 
Komiteemitglieder sind anerkannte Experten auf dem Gebiet der Usable Security and Privacy aus Wissenschaft und Praxis:

- Andreas Both (Unister GmbH, DE)

- Alexander De Luca (Google, $\mathrm{CH})$

- Simone Fischer-Hübner (Karlstad University, SE)

- Stefan Grünvogel (Fachhochschule Köln, DE)

- Marit Hansen (Unabhängiges Landeszentrum für Datenschutz Schleswig-Holstein, DE)

- Tobias Hirsch (Technische Universität Berlin, DE)

- Holger Junker (Bundesamt für Sicherheit in der Informationstechnik, DE)

- Michaela Kauer (Custom Interactions UG, DE)

- Peter Mayer (Technische Universität Darmstadt, DE)

- Sebastian Möller (Technische Universität Berlin, DE)

- Günter Müller (Universität Freiburg, DE)

Alle eingereichten Beiträge wurden durch die Mitglieder des Programmkomitees in einem Double-Blind-Peer-Review-Verfahren begutachtet. Auswahlkriterien für die Annahme waren die Relevanz, Originalität und wissenschaftliche Qualität des Beitrags, eine klare Beschreibung des Lösungsansatzes und ein überzeugender Beleg für dessen Nützlichkeit.

\section{Organisation und Durchführung}

Luigi Lo Iacono (Fachhochschule Köln, luigi.lo_iacono@fh-koeln.de)

Hartmut Schmitt (HK Business Solutions, schmitt@hk-bs.de)

Der Workshop „Usable Security and Privacy: Nutzerzentrierte Lösungsansätze zum Schutz sensibler Daten“ ist eine Veranstaltung des Verbundvorhabens USecureD ${ }^{1}$ in Zusammenarbeit mit der Fachgruppe E-Commerce und E-Government (FG ECOM) der Gesellschaft für Informatik.

\section{Danksagung}

Das Verbundvorhaben „USecureD - Usable Security by Design“ wird mit Mitteln des Bundesministeriums für Wirtschaft und Energie unter dem Förderkennzeichen 01MU14002 gefördert (Förderinitiative: Einfach intuitiv - Usability für den Mittelstand).

$1 \quad$ Vgl. dazu http://www.usecured.de 
